\title{
Factors associated with time to diagnosis from symptom onset in patients with early rheumatoid arthritis
}

\author{
Soo-Kyoung Cho ${ }^{1,2}$, Dam Kim, ${ }^{1,2}$, Soyoung Won ${ }^{2}$, Jiyoung Lee ${ }^{2}$, Chan-Bum Choi ${ }^{1,2}$, Jung-Yoon Choe ${ }^{3}$, \\ Seung-Jae Hong ${ }^{4}$, Jae-Bum Jun ${ }^{1}$, Tae-Hwan Kim ${ }^{1}$, Eunmi Koh', Hye-Soon Lee ${ }^{6}$, Jisoo Lee ${ }^{7}$, Dae-Hyun Yoo ${ }^{1}$, \\ Bo Young Yoon ${ }^{8}$, Sang-Cheol Bae ${ }^{1,2}$, Yoon-Kyoung Sung ${ }^{1,2}$, and the Korean Observational Study \\ Network for Arthritis (KORONA) Investigators
}

\begin{abstract}
Department of Rheumatology, ${ }^{1}$ Hanyang University Hospital for Rheumatic Diseases, Seoul; ${ }^{2}$ Clinical Research Center for Rheumatoid Arthritis (CRCRA), Seoul; ${ }^{3}$ Catholic University of Daegu School of Medicine, Daegu; ${ }^{4}$ Kyung Hee University Medical Center, Seoul; ${ }^{5}$ Samsung Medical Center, Sungkyunkwan University School of Medicine, Seoul; ${ }^{6}$ Hanyang University Guri Hospital, Guri; ${ }^{7}$ Ewha Womans University Mokdong Hospital, Seoul; ${ }^{8}$ Inje University Ilsan Paik Hospital, Goyang, Korea
\end{abstract}

Received: April 2, 2017

Revised : June 10, 2017

Accepted: June 20, 2017

\section{Correspondence to}

Yoon-Kyoung Sung, M.D.

Department of Rheumatology,

Hanyang University Hospital for

Rheumatic Diseases, 222-1

Wangsimni-ro, Seongdong-gu,

Seoul 04763 , Korea

Tel: +82-2-2290-9207

Fax: +82-2-2298-8231

E-mail:sungyk@hanyang.ac.kr

Sang-Cheol Bae, M.D.

Department of Rheumatology,

Hanyang University Hospital for

Rheumatic Diseases, 222-1

Wangsimni-ro, Seongdong-gu,

Seoul 04763 , Korea

Tel: $+82-2-2290-9237$

Fax: +82-2-2298-8231

E-mail: scbae@hanyang.ac.kr
Background/Aims: To identify the factors associated with time to diagnosis after symptom onset in patients with early rheumatoid arthritis (RA).

Methods: Early RA patients with $\leq 1$ year of disease duration in the KORean Observational study Network for Arthritis (KORONA) database were included in this analysis. Patients were further divided into two groups according to the time to diagnosis from symptom onset: the early diagnosis group (time to diagnosis $\leq 1$ year) and the late diagnosis group (time to diagnosis > 1 year). Using the multivariable regression model, we identified factors associated with early diagnosis.

Results: Among 714 early RA patients, 401 patients (56.2\%) and 313 patients (43.8\%) were included in the early diagnosis and late diagnosis groups, respectively. The mean disease duration was 0.47 years in the early diagnosis group and 0.45 years in the late diagnosis group. In multivariable model analysis, greater age at onset (odds ratio [OR], 1.03; 95\% confidence interval [CI], 1.02 to 1.05), high school education or higher (OR, 1.68; 95\% CI, 1.14 to 2.47), higher income (OR, 1.48; 95\% CI, 1.05 to 2.08 ), and initial small joint involvement (OR, 1.42; $95 \%$ CI, 1.02 to 1.98) were factors associated with early diagnosis. At diagnosis, disease activity scores using 28 joints on diagnosis $(3.81 \pm 1.44$ vs. $3.82 \pm 1.42, p=0.92)$ and functional disability $(0.65 \pm 0.61$ vs. $0.57 \pm 0.62, p=0.07)$ did not different between the two groups. However, hand joint erosion on X-ray ( $37.8 \%$ vs. $25.6 \%, p<0.01$ ) was more common in the late diagnosis group than the early diagnosis group.

Conclusions: Older onset age, higher educational level and income, and initial small joint involvement were positive factors for early diagnosis of RA.

Keywords: Early diagnosis; Arthritis, rheumatoid 


\section{INTRODUCTION}

Early diagnosis is an optimal target for better outcomes in rheumatoid arthritis (RA) in clinical practice $[1,2]$, because early treatment is associated with improved outcomes [2-4]. Furthermore, a UK National Audit Office report concluded that initial management in secondary care of adopting an early diagnosis approach was more cost-effective compared to general physician referral [5]. Despite this, delays occur between the patient seeking medical attention and assessment by a rheumatologist for treatment initiation. These delays occurred at several stages, including the time from symptom onset to assessment in primary care, primary care assessment to secondary care rheumatology referral, rheumatology referral to rheumatology assessment and rheumatology assessment to initiation of disease-modifying antirheumatic drugs (DMARDs) [6].

When considering interventions to address this problem, it is informative to account for the components of the patient journey that contribute to delayed diagnosis. Some factors in this journey have been shown to vary markedly across healthcare infrastructures in Europe [7]. For example, the delay of patients in seeking medical advice was an important factor in the UK [8], whereas the initial healthcare professional delay was significantly longer than the patient delay in Germany [7]. Therefore, the health environment, including the healthcare delivery system of the country, should be considered when investigating factors related to diagnosis delay. In addition, recent reports suggested that causes of treatment delay in early RA differ according to patient autoantibody status [9].

In order to identify factors related to delayed diagnosis of RA and thus facilitate early diagnosis, it would be helpful to determine the sociodemographic or clinical factors influencing recognition of this disease.

In this study, we aimed to identify factors associated with time to diagnosis after symptom onset in patients with early RA.

\section{METHODS}

\section{Study population}

Patients over the age of 18 years who satisfied the 1987
American College of Rheumatology (ACR) classification criteria for RA were recruited by rheumatologists in 23 centers across South Korea as part of the KORean Observational study Network for Arthritis (KORONA) [10]. The early RA cohort was composed of RA patients whose disease duration was 1 year or less among RA patients enrolled in KORONA. After exclusion of 17 patients without information about the date of symptom onset or RA diagnosis, 714 early RA patients were included in this analysis. All patients provided informed consent under the Institutional Review Board of Hanyang University Hospital-approved study protocol (HYUH 2009-04-003). The time to diagnosis was defined as the duration between symptom onset and diagnosis of RA for each patient. The early diagnosis group was defined based on a time to diagnosis of 1 year or less, while the late diagnosis group included all patients with a time to diagnosis > 1 year (Supplementary Fig. 1).

\section{Data collection}

All patients completed an initial questionnaire to establish their demographic profile, socio-economic status and disease-specific outcome. We collected RA-related information such as age at symptom onset, initial symptomatic joint, disease duration, rheumatoid factor (RF) status, anti-citrullinated protein antibody (ACPA), inflammatory markers and joint assessment performed by rheumatologists or well-trained health professionals. Data on family type, educational level, income and comorbidities categorized as cardiovascular disease including angina, myocardial infarction and cerebrovascular disease, hypertension, gastrointestinal disease including gastritis and peptic ulcer disease, and diabetes mellitus were collected.

\section{Statistical analysis}

We used descriptive statistics to compare the demographic and socioeconomic characteristics of the early diagnosis and late diagnosis groups. Chi-square tests were used for categorical variables and Student $t$ test was performed for continuous variables. Using univariable and multivariable analysis, we identified factors associated with early diagnosis. Further analysis was performed to identify the impact of age and gender factor on early diagnosis using a multivariable regression model. All analyses were performed using SAS version 
9.2 (SAS Institute Inc., Cary, NC, USA). Results were considered statistically significant when $p$ values were $<0.05$.

\section{RESULTS}

\section{Demographic and clinical features}

Among the 714 early RA patients, 401 patients (56.2\%) and 313 patients $(43.8 \%)$ were included in the early diagnosis and late diagnosis groups, respectively (Fig. 1). Mean time to diagnosis was $1.89 \pm 3.37$ years; $0.51 \pm 0.34$ years in the early diagnosis group and $3.66 \pm 4.50$ years in the late diagnosis group.

The clinical and demographic characteristics of the study population are presented in Table 1. All patients were enrolled at a very early stage; their mean disease duration was defined as the period between diagnosis

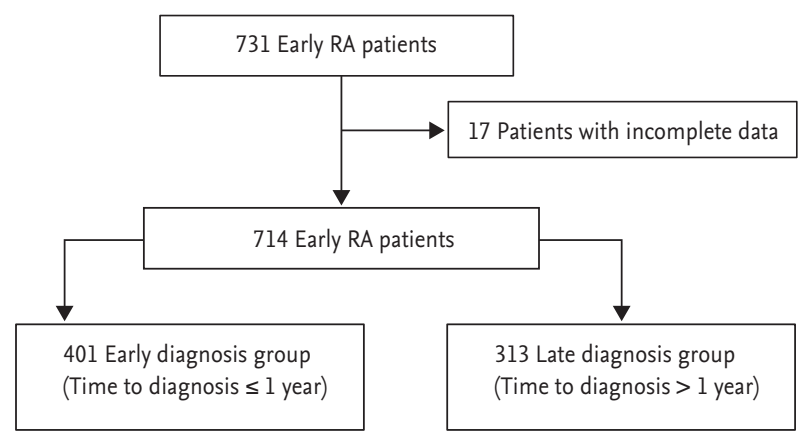

Figure 1. Patient selection flow. RA, rheumatoid arthritis.

Table 1. Differences in demographic and clinical characteristics between early diagnosis group and late diagnosis group

\begin{tabular}{|c|c|c|c|c|}
\hline Variable & $\operatorname{Total}(\mathrm{n}=714)$ & $\begin{array}{l}\text { Early diagnosis group } \\
(\mathrm{n}=401)\end{array}$ & $\begin{array}{l}\text { Late diagnosis group } \\
(\mathrm{n}=313)\end{array}$ & $p$ value \\
\hline Age, yr & $50.9 \pm 12.7$ & $50.4 \pm 12.8$ & $51.5 \pm 12.6$ & 0.27 \\
\hline Onset age, yr & $48.6 \pm 12.7$ & $49.5 \pm 12.8$ & $47 \cdot 4 \pm 12.6$ & 0.03 \\
\hline Female sex & $562(78.7)$ & $318(79 \cdot 3)$ & $244(78.0)$ & 0.73 \\
\hline Time to diagnosis, $\mathrm{yr}^{\mathrm{a}}$ & $1.89 \pm 3.37$ & $0.51 \pm 0.34$ & $3.66 \pm 4.50$ & $<0.01$ \\
\hline Disease duration, $\mathrm{yr}^{\mathrm{b}}$ & $0.47 \pm 0.30$ & $0.48 \pm 0.30$ & $0.45 \pm 0.29$ & 0.22 \\
\hline Family history of RA & $83(12.2)$ & $43(11.3)$ & $40(13.4)$ & 0.48 \\
\hline Level of education & & & & 0.09 \\
\hline Middle school or less & $279(39.1)$ & $145(36.3)$ & $134(42.8)$ & \\
\hline High school or more & $434(60.9)$ & $255(63.8)$ & $179(57.2)$ & \\
\hline Monthly income, US dollar & & & & 0.04 \\
\hline$<1,900$ & $311(43 \cdot 6)$ & $161(40.2)$ & $150(48.1)$ & \\
\hline$\geq 1,900$ & $402(56.4)$ & $240(59.9)$ & $162(51.9)$ & \\
\hline First symptom in small joint & $505(70.7)$ & $295(73 \cdot 6)$ & $210(67.1)$ & 0.07 \\
\hline No. of comorbidity & & & & 0.76 \\
\hline $\mathrm{o}$ & $331(46.4)$ & $181(45 \cdot 1)$ & $150(47 \cdot 9)$ & \\
\hline 1 & $240(33.6)$ & $138(34.4)$ & $102(32.6)$ & \\
\hline$\geq 2$ & $143(20.0)$ & $82(20.5)$ & $61(19 \cdot 5)$ & \\
\hline RF positivity & $638(89.4)$ & $361(90.0)$ & $277(88.5)$ & 0.59 \\
\hline ACPA positivity & $570 / 666(85.6)$ & $315(84.9)$ & $255(86.4)$ & 0.65 \\
\hline DAS28-ESR & $3.82 \pm 1.43$ & $3.81 \pm 1.44$ & $3.82 \pm 1.42$ & 0.92 \\
\hline HAQ-DI score & $0.60 \pm 0.61$ & $0.57 \pm 0.62$ & $0.65 \pm 0.61$ & 0.07 \\
\hline Hand joint erosion on X-ray & $192(31.0)$ & $88(25.6)$ & $104(37.8)$ & $<0.01$ \\
\hline
\end{tabular}

Values are presented as mean $\pm \mathrm{SD}$ or number (\%).

RA, rheumatoid arthritis; RF, rheumatoid factor; ACPA, anti-citrullinated protein antibody; DAS28-ESR, disease activity score 28 joints-erythrocyte sedimentation ratio; HAQ-DI, health assessment questionnaires-disability index.

${ }^{\mathrm{a}} \mathrm{The}$ period from symptom onset to diagnosis of disease.

${ }^{b_{T}}$ The period from diagnosis of disease to assessment for this study. 
Cho SK, et al. Time to diagnosis from symptom onset in rheumatoid arthritis

Table 2. Possible factors associated with early diagnosis in early RA patients

\begin{tabular}{|c|c|c|}
\hline Variable & Univariable analysis & Multivariable analysis \\
\hline Onset age, yr & $1.01(1.00-1.03)$ & $1.03(1.02-1.05)$ \\
\hline Female sex & $1.08(0.76-1.55)$ & $1.25(0.86-1.84)$ \\
\hline Family history of RA & $0.82(0.52-1.31)$ & - \\
\hline \multicolumn{3}{|l|}{ Education } \\
\hline Middle school or less & Reference & Reference \\
\hline High school or more & $1.32(0.97-1.78)$ & $1.68(1.14-2.47)$ \\
\hline \multicolumn{3}{|l|}{ Monthly income, US dollar } \\
\hline$<1,900$ & Reference & Reference \\
\hline$\geq 1,900$ & $1.38(1.02-1.86)$ & $1.48(1.05-2.08)$ \\
\hline \multicolumn{3}{|l|}{ Body mass index, $\mathrm{kg} / \mathrm{m}^{2}$} \\
\hline$<18.5$ & $0.74(0.38-1.42)$ & - \\
\hline$\geq 18.5$ and $<23.0$ & Reference & - \\
\hline$\geq 23.0$ & $0.75(0.55-1.02)$ & - \\
\hline Regular exercise & $1.32(0.97-1.78)$ & - \\
\hline First symptom in small joint & $1.37(0.99-1.89)$ & $1.42(1.02-1.98)$ \\
\hline \multicolumn{3}{|l|}{ No. of comorbidities } \\
\hline 0 & - & Reference \\
\hline 1 & $1.12(0.80-1.57)$ & $0.99(0.69-1.41)$ \\
\hline$\geq 2$ & $1.11(0.75-1.66)$ & $0.95(0.62-1.45)$ \\
\hline Rheumatoid factor positivity & $1.17(0.73-1.89)$ & $1.24(0.76-2.04)$ \\
\hline ACPA positivity & $0.88(0.57-1.37)$ & - \\
\hline
\end{tabular}

Values are presented as multi-adjusted odds ratio ( $95 \%$ confidence interval).

RA, rheumatoid arthritis; ACPA, anti-citrullinated protein antibody.

Table 3. Possible factors associated with early diagnosis according to onset age

\begin{tabular}{|c|c|c|}
\hline Variable & $\begin{array}{l}\text { Younger onset group } \\
(<60 \text { years })(n=568)\end{array}$ & $\begin{array}{l}\text { Elderly onset group } \\
(\geq 60 \text { years })(n=146)\end{array}$ \\
\hline Onset age, yr & $1.03(1.01-1.05)$ & $1.02(0.95-1.10)$ \\
\hline Female sex & $1.47(0.94-2.29)$ & $0.80(0.36-1.78)$ \\
\hline \multicolumn{3}{|l|}{ Education } \\
\hline Middle school or less & Reference & Reference \\
\hline High school or more & $1.66(1.07-2.58)$ & $1.43(0.60-3.38)$ \\
\hline \multicolumn{3}{|l|}{ Monthly income, US dollar } \\
\hline$<1,900$ & Reference & Reference \\
\hline$\geq 1,900$ & $1.47(1.01-2.15)$ & $1.72(0.74-4.01)$ \\
\hline Initial small joint involvement & $1.33(0.91-1.93)$ & $1.93(0.93-4.01)$ \\
\hline \multicolumn{3}{|l|}{ No. of comorbidities } \\
\hline o & Reference & Reference \\
\hline 1 & $1.07(0.73-1.59)$ & $0.66(0.26-1.66)$ \\
\hline$\geq 2$ & $0.86(0.53-1.39)$ & $0.98(0.37-2.60)$ \\
\hline Rheumatoid factor positivity & $1.20(0.70-2.08)$ & $1.52(0.46-4.99)$ \\
\hline
\end{tabular}

Values are presented as multi-adjusted odds ratio ( $95 \%$ confidence interval). 
and enrollment in this study was less than 6 months ( $0.47 \pm 0.30$ years). The onset age of the early diagnosis group was older than the late diagnosis group $(49.5 \pm 12.8$ vs. $47.4 \pm 12.6, p=0.03$ ). The proportion of females in both groups was similar (79.3\% vs. $78.0 \%, p=0.73$ ). The early diagnosis group had a higher income $(59.9 \%$ with income $\geq 1,900$ US dollar/mon; exchange rate 1 US dollar $=1,000$ Korean won) than the late diagnosis group (51.9\%, $p=0.04)$. Hand joint erosion on X-ray was more common in the late diagnosis group (37.8\%) than the early diagnosis group $(25.6 \%, p<0.01)$. The level of education was also higher in the early diagnosis group than the late diagnosis group, but this was without statistical significance $(63.8 \%$ vs. $57.2 \%$ of patients with high school education or more, $p=0.09)$. Family history of RA was not different between the two groups. DAS28-ESR and functional disability were not different between the two groups.

\section{Factors associated with early diagnosis}

Older age at symptom onset (odds ratio [OR], 1.03; 95\% confidence interval [CI], 1.02 to 1.05), higher level of education (OR, 1.68; CI, 1.14 to 2.47), higher income (OR, 1.48; $\mathrm{CI}, 1.05$ to 2.08$)$ and initial small joint involvement (OR, 1.42; CI, 1.02 to 1.98) were identified as factors associated with early diagnosis in patients with early RA (Table 2 ).

\section{Factors associated with early diagnosis according to onset age}

We divided early RA patients into two groups: the elderly onset group whose onset age was 60 years or older and the young onset group aged less than 60 years at onset. In the younger onset group, older age at symptom onset (OR, 1.03; CI, 1.01 to 1.05), higher level of education (OR, 1.66; CI, 1.07 to 2.58) and higher income (OR, 1.47; CI, 1.01 to 2.15 ) were predictive factors for early diagnosis. We could not identify any predictive factors in the elderly onset group (Table 3).

\section{DISCUSSION}

The average delay in the assessment of patients with RA by rheumatologists in Korea was 1.89 years. This delay in RA diagnosis was longer than that reported across Europe (from 16 weeks in Austria to 38 weeks in Greece)
[7], but shorter than that in Saudi Arabia (30 months) [11]. Our study suggested that old age at symptom onset, higher education level or income, and initial small joint involvement were factors associated with shorter time to diagnosis in early RA patients.

In Korea, the time lag between the patient seeking medical advice and a rheumatologist seeing the patient is typically not long. In the Korean healthcare delivery system, the patients have freedom of choice and can go to any doctor or any medical institution, including any hospital that they choose. Furthermore, all people in South Korea are eligible for coverage under the National Health Insurance Program [12]. Therefore, the main component of delayed diagnosis in Korea may be patient-dependent. Based on the results of our study, patients with younger onset age and without involvement of the small joints are more likely to have a delay in RA diagnosis. This may be related to public communication and education regarding typical RA symptoms. The factors related to RA disease process were consistent with previous reports $[13,14]$. There is controversy over the relationship between presence of autoantibody in patients and delayed RA diagnosis. In our study, positivity of RF or ACPA was not related to early diagnosis. However, a previous report suggested the significance of RF for early diagnosis [14] and in a recent report, positivity of RF and positivity of ACPA were both associated with a significant delay in initiation of DMARDs [9]. In addition, delayed diagnosis can result from the fact that identification and diagnosis of RA is difficult due to the insidious nature of the disease and the widespread use of non-steroidal anti-inflammatory drugs, which have the potential to mask RA symptoms.

An American College of Rheumatology-European League Against Rheumatism (ACR-EULAR) task force suggested that delays to diagnosis and starting treatment include four main stages: (1) from symptom onset in patient to assessment in primary care; (2) from primary care provider to rheumatology referral; (3) from rheumatology referral to assessment; and (4) from rheumatology assessment to commencement of DMARDs therapy [6]. Previous systematic literature reviews showed various efforts at addressing referral delays [6]. For the first two steps, internet and website information based on patient self-administered questionnaires to recognize patients with possible inflammatory arthritis were included [14- 
16]. The data in a previous report were restricted to Europe and North America. In Korea we also established an internet website (www.rheusearch.com) based on a self-administered early inflammatory arthritis detection tool [15] adapted to the Korean language, thus allowing the patient to evaluate the possibility of early inflammatory arthritis by themselves. This guide may improve the accessibility of patients with inflammatory arthritis to a rheumatologist and help to lead to early diagnosis of rheumatic diseases in Korea.

Early diagnosis of RA is associated with decreased radiologic progression or functional disability [4,17]. In our study, the frequency of hand joint erosion was lower in the early diagnosis group than in the late diagnosis group, although their disease durations were similar.

Our study has some limitations. First, our data cannot separate the delay in the time from symptom onset to seeing the primary physician and the time from visiting the primary physician to seeing a rheumatologist. Therefore, we cannot determine the delay period associated with primary physician referral to a specialist. However, it is more important to improve the early detection of inflammatory arthritis in the patients' perspective in Korea because patients feeling pain in their joints can see a rheumatologist in the primary care setting in the Korean health environment. Second, the reported symptom onset date might be inaccurate because of recall bias. To avoid this bias, we extracted early RA patients who were enrolled within 1 year from RA diagnosis. Third, it is possible that not all factors influencing the delay in RA diagnosis were included in this analysis. However, our finding of patient factors associated with delayed diagnosis can be important to generate a strategy for the first delay step from symptom onset to assessment in primary care.

The findings of our study are noteworthy in several ways. First, the reported delay in RA diagnosis was also present in Korea, which is known to have good healthcare access. Second, we found that patients with low educational level, young age or initial involvement of the large joints are at high risk for delayed diagnosis of RA. This result implies that we have focused on patients with typical characteristics or symptoms of RA to date. However, it needs to focus for the education of young patients and primary physicians.

In conclusion, we identified a delay in RA diagnosis in Korea, consistent with findings from various regions around the world. Older age at symptom onset, higher education level or income, and initial involvement of the small joints were factors associated with a shorter time to diagnosis in early RA patients. Our efforts to improve the accessibility of patients with inflammatory arthritis to rheumatologists could accelerate early RA diagnosis.

\section{KEY MESSAGE}

1. Our data showed a delay in rheumatoid arthritis (RA) diagnosis in Korea, which is known to have good healthcare access.

2. Older onset age, higher educational level and income, and initial small joint involvement were positive factors for early diagnosis of RA patients.

\section{Conflict of interest}

No potential conflict of interest relevant to this article was reported.

\section{Acknowledgments}

The authors wish to acknowledgee the assistance of the following investigators who enrolled patients in the KORean Observational study Network for Arthritis (KORONA).

This research was supported by a grant of the Korea Health Technology R\&D Project through the Korea Health Industry Development Institute (KHIDI), funded by the Ministry of Health and Welfare, Korea (HI16Coo61).

\section{REFERENCES}

1. van der Linden MP, le Cessie S, Raza K, et al. Long-term impact of delay in assessment of patients with early arthritis. Arthritis Rheum 2010;62:3537-3546.

2. Combe B, Landewe R, Lukas C, et al. EULAR recommendations for the management of early arthritis: report of a task force of the European Standing Committee for International Clinical Studies Including Therapeutics (ESCISIT). Ann Rheum Dis 2007;66:34-45.

3. Emery P. Treatment of rheumatoid arthritis. BMJ 
2006;332:152-155.

4. Kim D, Choi CB, Lee J, et al. Impact of early diagnosis on functional disability in rheumatoid arthritis. Korean J Intern Med 2017;32:738-746.

5. National Audit Office. Services for people with rheumatoid arthritis [Internet]. London: National Audit Office, 2011 [cited 2017 Sep 25]. Available from: http://www.nao. org.uk/publications/0809/rheumatoid_arthritis.aspx.

6. Villeneuve E, Nam JL, Bell MJ, et al. A systematic literature review of strategies promoting early referral and reducing delays in the diagnosis and management of inflammatory arthritis. Ann Rheum Dis 2013;72:13-22.

7. Raza K, Stack R, Kumar K, et al. Delays in assessment of patients with rheumatoid arthritis: variations across Europe. Ann Rheum Dis 2011;70:1822-1825.

8. Kumar K, Daley E, Carruthers DM, et al. Delay in presentation to primary care physicians is the main reason why patients with rheumatoid arthritis are seen late by rheumatologists. Rheumatology (Oxford) 2007;46:1438-1440.

9. Pratt AG, Lendrem D, Hargreaves B, Aslam O, Galloway JB, Isaacs JD. Components of treatment delay in rheumatoid arthritis differ according to autoantibody status: validation of a single-centre observation using national audit data. Rheumatology (Oxford). 2016;55:1843-1848.

10. Sung YK, Cho SK, Choi CB, et al. Korean Observational Study Network for Arthritis (KORONA): establishment of a prospective multicenter cohort for rheumatoid arthritis in South Korea. Semin Arthritis Rheum 2012;41:745-751.

11. Hussain W, Noorwali A, Janoudi N, et al. From symptoms to diagnosis: an observational study of the journey of rheumatoid arthritis patients in Saudi Arabia. Oman Med J. 2016;31:29-34.

12. Song YJ. The South Korean health care system. Japan Med Assoc J 2009;52:206-209.

13. Chan KW, Felson DT, Yood RA, Walker AM. The lag time between onset of symptoms and diagnosis of rheumatoid arthritis. Arthritis Rheum 1994;37:814-820.

14. Hwang AS, Gall V, Liang MH. Evaluation of the internet for finding persons with undiagnosed rheumatoid arthritis and systemic lupus erythematosus. J Clin Rheumatol 2009;15:218-222.

15. Bell MJ, Tavares R, Guillemin F, Bykerk VP, Tugwell P, Wells GA. Development of a self-administered early inflammatory arthritis detection tool. BMC Musculoskelet Disord 2010;11:50.

16. Callahan LF, Pincus T. A clue from a self-report questionnaire to distinguish rheumatoid arthritis from noninflammatory diffuse musculoskeletal pain: the P-VAS:DADL ratio. Arthritis Rheum 1990;33:1317-1322.

17. Kyburz D, Gabay C, Michel BA, Finckh A; physicians of SCQM-RA. The long-term impact of early treatment of rheumatoid arthritis on radiographic progression: a population-based cohort study. Rheumatology (Oxford) 2011;50:1106-1110. 
Cho SK, et al. Time to diagnosis from symptom onset in rheumatoid arthritis

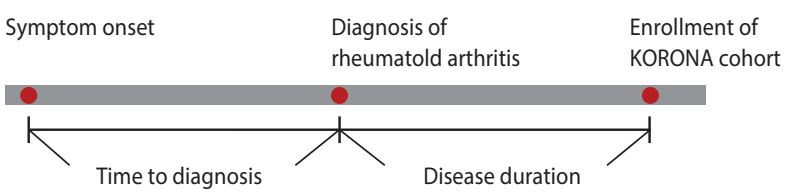

Supplementary Figure 1. The definitions of disease duration and time to diagnosis. KORONA, KOrean Observational Study Network for Arthritis. 